\title{
"Zeta" di frontiera: confronto tra giovani bolzanini italofoni e germanofoni
}

\section{Chiara Meluzzi}

\section{(2) OpenEdition \\ 12 Journals}

Edizione digitale

URL: http://journals.openedition.org/esp/666

DOI: $10.4000 /$ esp.666

ISSN: 2532-0319

Editore

Centre d'Information sur l'Éducation Bilingue et Plurilingue

\section{Edizione cartacea}

Data di pubblicazione: 1 dicembre 2015

Paginazione: 89-100

ISSN: 1127-266X

Notizia bibliografica digitale

Chiara Meluzzi, «"Zeta" di frontiera: confronto tra giovani bolzanini italofoni e germanofoni », Éducation

et sociétés plurilingues [Online], 39 | 2015, Messo online il 01 octobre 2016, consultato il 01 mai 2019.

URL : http://journals.openedition.org/esp/666 ; DOI : 10.4000/esp.666 


\section{"ZETA" DI FRONTIERA: CONFRONTO TRA GIOVANI BOLZANINI ITALOFONI E GERMANOFONI}

\section{Chiara MeLuZzI}

Nel peculiare contesto sociolinguistico offerto dall'Alto Adige/Südtirol, l'apprendimento della lingua "altra" rispetto alla propria L1 si trova a dover fare $i$ conti con due fattori: da un lato l'altra lingua (tedesco o italiano) viene insegnata come L2 fin dalle elementari, dall'altro per molti alunni questa lingua "altra" rischia di diventare una vera e propria LS, date le scarse occasioni non solo di contatto con l'altro gruppo linguistico quanto di uso della varietà di lingua appresa. Ciò è particolarmente avvertito dalla comunità italofona, dal momento che l'insegnamento del tedesco standard (Hochdeutsch) impartito nelle scuole italiane si scontra con l'effettivo uso da parte della comunità linguistica germanofona di varietà di tedesco ascrivibili al bavarese meridionale. Il complesso repertorio linguistico risulta inoltre complicata dalle dinamiche di maggioranza-minoranza linguistica. La situazione sociolinguistica diventa però più interessante qualora si vada a osservare anche micro-aspetti della produzione linguistica, quale appunto può essere considerata la variazione all'interno dello stesso parlante e tra parlanti diversi nella pronuncia di un singolo fono. In questo studio si intende pertanto proporre proprio una analisi di micro-variazione sulla pronuncia delle affricate dentali italiane /ts $\mathrm{dz} /$, due fonemi sulla cui realizzazione non incide tanto l'idea di una "norma" non pienamente specificata quanto aspetti sociolinguistici diversi legati, ad esempio, all'età e al genere del parlante, nonché alle sue onigini. Cosa ancora più interessante, questa stessa variazione trova un parallelo interessante anche nelle produzioni dei germanofoni, offrendosi dunque come implicita riflessione sul ruolo svolto non solo dalla scuola ma anche dal contatto linguistico nello sviluppo di alcune peculiarità fonetiche nella L2.

Parole chiave: bilinguismo, contatto linguistico, sociolinguistica, fonetica.

Dans le contexte sociolinguistique particulier du Tyrol septentrional, l'acquisition et l'enseignement d'une langue différente de la L1 sont liés à divers facteurs: l'autre langue (allemande ou italien) est apprise comme L2 dès l'entrée à l'école, mais pour beaucoup d'élèves, elle est une sorte de LS, puisqu'il y a peu d'occasions de contact avec l'autre groupe linguistique, et encore moins de possibilités d'employer réellement la variété apprise. Le groupe linguistique italien s'en plaint régulièrement, puisque la variété standard de l'allemand apprise à l'école n'est pas celle employée par les locuteurs d'allemand en Tyrol septentrional, qui parlent diverses variétés/dialectes du bavarois du sud. Ce répertoire linguistique se complique encore par les dynamiques complexes entre langues majonitaires et minoritaires. La variation sociolinguistique est très intéressante quand on observe quelque petit trait comme par exemple la variation dans la production d'un seul phonème par le même locuteur ou entre groupes de locuteurs. Dans cette étude, la micro-variation dans la réalisation des affriquées dentales de l'italien /ts dz/ sera envisagée par rapport aux variables sociolinguistiques telles que l'âge ou le sexe. Qui plus est, cette variation chez les locuteurs de l'italien L1 se trouve en parallèle avec les locuteurs de l'allemand, ainsi ouvrant le débat sur le rôle non seulement de l'enseignement mais aussi du contact lorsqu'on travaille sur un trait phonétique spécifique de la langue cible.

Mots-clés: bilinguisme, contact linguistique, sociolinguistique, phonétique. 
In the particular sociolinguistic context of South Tyrol, acquiring and teaching a language different from the L1 are linked to different factors: the other language (i.e., German or Italian) is taught as L2 since the beginning of school, but, on the other hand, for many pupils this "other" language becomes a sort of $L S$, since there are very few occasions of contact with the other linguistic group, and even fewer possibilities to actually use the variety learned. This is a typical complaint within the Italian linguistic group, since the standard variety of German learned in school is not the one used by the German speakers of South Tyrol, who speak different varieties/dialects of Southern Bavarian. This linguistic repertoire is also complicated by the not-so-easy dynamics between majority and minority languages. The sociolinguistic variation is even more interesting when observing some little aspects of linguistic production, as e.g. the variation in the production of a single phone by the same speaker or between groups of speakers. In this study, the micro-variation in the realization of Italian dental affricates /ts $d z$ / will thus be considered with respect to sociolinguistic variables such as speaker's age or sex. More interestingly, this variation among Italian L1 speakers will establish a parallel wirth German speakers, thus questioning the role not only of teaching but also of contact when developing some peculiar phonetic features of the target language.

Key-words: bilingualism, language contact, sociolinguistics, phonetics.

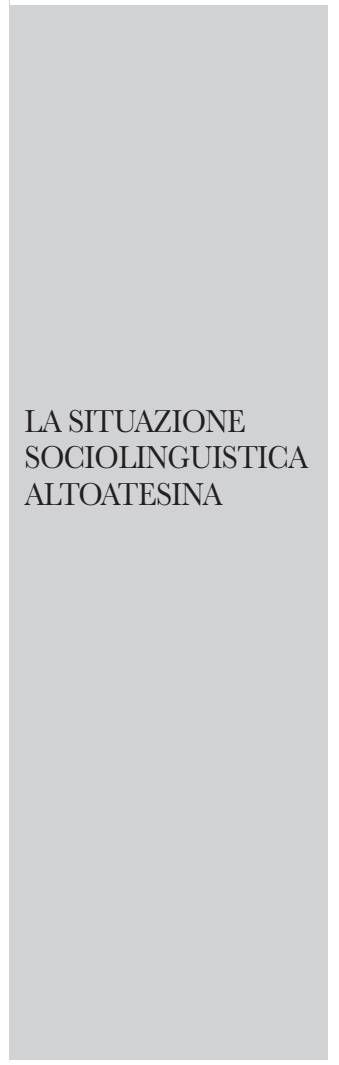

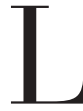

a situazione linguistica altoatesina offre interessanti spunti di indagine linguistica a diversi livelli di analisi. In particolare in questo lavoro si osserverà come il contatto linguistico tra giovani altoatesini appartenenti ai due principali gruppi linguistici (italiano e tedesco) si rifletta nella pronuncia di due particolari fonemi, ossia le affricate dentali /ts dz/. Saranno quindi osservate le influenze reciproche tra la pronuncia degli italofoni e dei tedescofoni, quindi in una varietà di italiano $\mathrm{L} 1 \mathrm{e}$ in una particolare varietà di L2.

Litaliano altoatesino ha una particolare connotazione, legata alla singolare storia sociolinguistica della regione dell'Alto Adige/Südtirol, appartenente allo stato italiano solo dal 1918. Alla popolazione germanofona si andò ad aggiungere, a partire dagli anni '20, un altissimo numero di italofoni, secondo un piano preciso di italianizzazione della regione ad opera del regime fascista (cfr. Petri 1989). I nuovi arrivati provenivano inizialmente dalle vicine aree trentine e venete, in particolare dalle zone di Rovigo e Padova: si trattava per la maggior parte di contadini che si spostavano nelle grandi città, in particolare a Bolzano, per venire impiegati come operai nella fiorente industria altoatesina. Non mancavano tuttavia anche impiegati e funzionari da Milano e Roma, inviati in Alto Adige con lo scopo di sostituire il personale tedescofono. Infine, dopo la seconda guerra mondiale l'intera zona fu interessata da una forte migrazione anche dalle regioni meridionali d'Italia e, in particolare, dalla Campania e dalla Calabria. A livello sociolinguistico, inoltre, si può notare come i più giovani italofoni rappresentino sempre una seconda se non una terza generazione d'immigrazione, conservando anche in misura minore gli usi linguistici delle regioni d'origine dei propri genitori o dei propri nonni; si noti però che la presenza di un influsso dialettale sull'italiano di Bolzano è ancora oggi questione non sufficientemente indagata e con numerosi 
"Zeta" di frontiera: confronto tra giovani bolzanini italofoni e germanofoni

C. Meluzzı spunti di discussione (cfr. Meluzzi 2015).

Va da sé che questo massiccio afflusso di italofoni modificò profondamente la facies linguistica dell'Alto Adige. Ancora oggi permane una profonda differenza in particolare tra le città, a maggioranza italofone, e le campagne, sempre a maggioranza tedescofona, come dimostrano anche gli ultimi dati del censimento nazionale della popolazione del 2011 (cfr. Astat 2012). Sono inoltre da ricordare le tensioni, ancora oggi in parte esistenti, tra i due principali gruppi linguistici, che portarono negli anni '60 e negli anni '80 addirittura ad attentati dinamitardi in quella che è conosciuta come la stagione delle bombe (cfr. Baur et al. 2008).

A livello legislativo, dal 1972 è in vigore in Alto Adige il cosiddetto "Pacchetto", ossia il Secondo Statuto di Autonomia, che riconosce nella regione tre lingue ufficiali, ossia italiano, tedesco e ladino. Da quella data è inoltre in vigore il sistema di divisione dei posti di lavoro nella sfera pubblica in modo proporzionale tra i tre gruppi linguistici. La composizione di questi ultimi è valutata ogni dieci anni attraverso la compilazione, da parte dei cittadini altoatesini, di una auto-dichiarazione di appartenenza linguistica nel corso del censimento nazionale della popolazione. Tra le altre importanti riforme introdotte dal "Pacchetto" vi è inoltre l'insegnamento della seconda lingua a partire dalla scuola elementare (si vedano in proposito le considerazioni di Freddi 1982). Gli scambi tra gruppo italiano e gruppo tedesco, tuttavia, sono stati fortemente limitati, almeno fino agli ultimi anni, durante $\mathrm{i}$ quali si sta assistendo a diverse iniziative anche a livello scolastico volte a stabilire maggiori contatti tra i due gruppi linguistici (cfr. Baur et al. 2008). Inoltre, come rilevano Abel, Vettori e Forer (2010), all'interno del complesso repertorio sociolinguistico altoatesino vanno considerate anche le dinamiche di maggioranza-minoranza linguistica, che si evidenziano in primo luogo negli atteggiamenti linguistici dei parlanti verso la propria e l'altra lingua (cfr. Meluzzi 2014). Ciò nonostante, si può evidenziare come le generazioni più giovani stiano avendo la possibilità di un maggiore contatto con i parlanti dell'altro gruppo linguistico, a differenza di quanto era avvenuto per le generazioni precedenti, in contesti anche extra-scolastici. La necessità di un maggiore contatto linguistico è particolarmente avvertito dal gruppo italofono, specialmente se residente in quei quartieri della città di Bolzano sorti durante il periodo di italianizzazione forzata della regione (cfr. Meluzzi 2010 e 2014).

Per questo motivo nel presente contributo ci si concentrerà sugli usi linguistici dei giovani altoatesini, prendendo in considerazione la pronuncia delle affricate dentali italiane, in un campione di parlanti sia italofoni sia germanofoni. La scelta di questa variabile linguistica è 
"Zeta" di frontiera: confronto tra giovani bolzanini italofoni e germanofoni

C. Meluzzı stata dettata dalla alta variabilità diatopica di questi fonemi nelle varietà regionali e nei dialetti italiani (cfr. Loporcaro 2009), in particolare in quelli che hanno caratterizzato la storia dell'italofonia bolzanina. Inoltre, si ricorda che anche il tedesco possiede nel suo inventario fonologico l'affricata dentale sorda /ts/, ma la sua natura monooppure bifonematica è ancor oggi oggetto di discussione (si veda in proposito Luschützky 1992). Questo tipo di variabile è dunque un interessante punto di partenza per indagare non solo la possibile emergenza di una varietà nuova di italiano altoatesino (cfr. Meluzzi 2013), ma anche le possibili influenze dovute a contatto tra gruppo linguistico italofono e gruppo linguistico germanofono. Infatti, il confronto tra i giovani parlanti dei due gruppi linguistici mostrerà come effetti dovuti a contatto si possano rintracciare tanto a livello di L1 quanto a livello di L2.

L'approccio metodologico che si è scelto per questo tipo di indagine è di tipo sociofonetico, andando ad osservare se e in che misura la micro-variazione fonetica possa assumere significato sociale (cfr. Foulkes et al. 2010). La caratteristica principale della ricerca in campo sociofonetico concerne in particolare la raccolta e l'analisi dei dati. Si adotterà pertanto un approccio il più possibile multidimensionale, con l'obiettivo di indagare la complessa interazione tra pattern diversi di variabili sia linguistiche sia sociali. A questo si associa la volontà di prendere in considerazione fenomeni non solo discreti ma anche continui, andando quindi a preferire una analisi di grana fine dei dati linguistici e una gradualità nella distribuzione dei fenomeni osservati (cfr. Foulkes et al. 2010).

Nel condurre un'analisi di tipo sociofonetico uno dei problemi principali riguarda la qualità acustica dei dati elicitati. Per questo motivo si tende solitamente a effettuare registrazioni in contesti molto controllati, pur se in tal caso risulterà molto difficile accedere ai livelli più informali, in senso laboviano (cfr. Labov 1994), del sistema linguistico. Nel nostro caso, la volontà di offrire una prima indagine sull'italiano parlato a Bolzano ha portato chi scrive ad utilizzare una lista di parole, comprendente 310 item, di cui 66 contenenti una affricata dentale. I file audio, in formato .wav, sono stati acquisiti tramite registratore ZOOM H2 e microfono esterno Sony ECM-MS957.

Rispetto al corpus utilizzato per la tesi di dottorato di chi scrive (cfr. Meluzzi 2013), per la presente analisi sono stati utilizzati 8 parlanti, 4 tedescofoni e 4 italofoni, che condividessero le medesime caratteristiche sociolinguistiche, come mostrato in tabella 1. Si tratta dunque di parlanti giovani (18-30 anni), divisi in egual numero tra maschi e femmine, tutti di livello di istruzione alto (diploma superiore, laurea). Per 
"Zeta" di frontiera: confronto tra giovani bolzanini italofoni e germanofoni

C. Meluzzı quanto riguarda il gruppo italofono, inoltre, sono stati considerati solo parlanti che appartenessero alla terza generazione di immigrazione in Alto Adige, ossia che avessero entrambi i genitori già nati a Bolzano e provincia.

\begin{tabular}{|l|l|l|l|l|}
\hline NOME & GRUPPO & SESSO & ETA & TIT. STUDIO \\
\hline 001GC & ITA & F & 19 & Diploma \\
\hline 011StLG & ITA & F & 31 & Diploma \\
\hline 018ALG & ITA & M & 26 & Laurea \\
\hline 040AS & ITA & M & 23 & Diploma \\
\hline 02HAN & TED & M & 25 & Laurea \\
\hline 03FRA & TED & F & 25 & Laurea \\
\hline 04LIS & TED & F & 24 & Laurea \\
\hline 05ALE & TED & M & 24 & Diploma \\
\hline
\end{tabular}

Tab. 1. Scheda sociolinguistica dei parlanti del corpus.

Il corpus così composto consta di 526 tokens contenenti una affricata dentale estratti dalla lista di parole, da cui risultano già esclusi due casi relativi alla parlante $001 \mathrm{GC}$ per problemi di audio. Tutti i tokens sono stati annotati sul software di analisi acustica PRAAT (cfr. Boersma \& Weenik 2013), seguendo un protocollo appositamente creato per l'annotazione di questi foni (cfr. Meluzzi 2013: 65-75), che ha permesso l'estrazione automatica dei valori di durata e di luogo di articolazione.

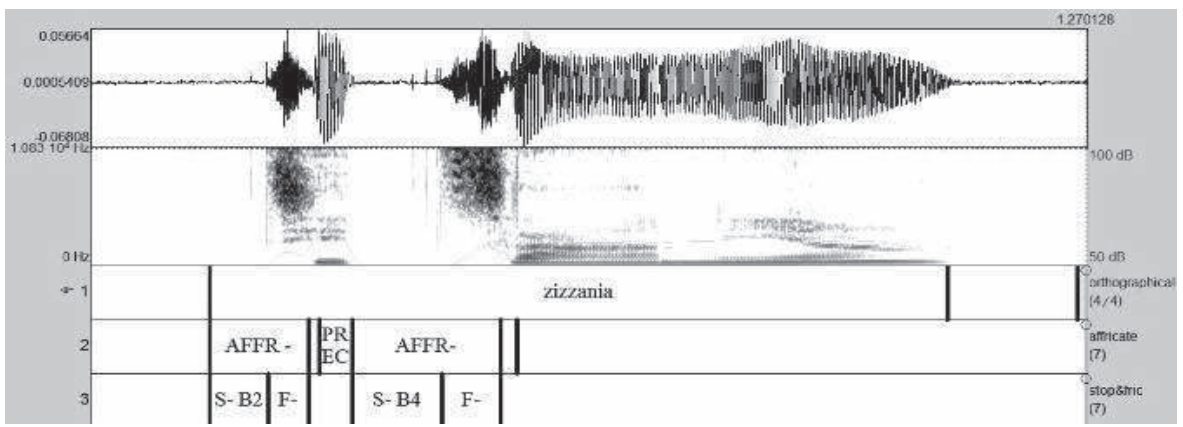

Fig. 1. Esempio di annotazione di una affricata dentale prodotto da 05ALE. In figura 1 si riporta un esempio di annotazione delle due affricate dentali contenute nella parola zizzania da parte di un parlante tedescofono (05ALE). Si può apprezzare l'assenza della barra di sonorità alle basse frequenze dello spettrogramma, che caratterizza pertanto entrambi i 
"Zeta" di frontiera: confronto tra giovani bolzanini italofoni e germanofoni

C. Meluzz!

ANALISI

E DISCUSSIONE DEI DATI foni come sordi (tag "AFFR -"). Si individuano inoltre agevolmente numerosi scoppi (burst) della parte occlusiva, visibili in particolar modo sulla forma d'onda e annotati nella terza riga ("B2 - B4"). L'individuazione dei due momenti occlusivo e fricativo è resa possibile sia dalla visualizzazione dell'inizio dell'aperiodicità diffusa alle alte frequenze tipica della produzione di una fricativa, sia dal contemporaneo innalzamento dell'intensità subito dopo lo scoppio dell'occlusiva. Per l'esempio fornito, si precisa che alla seconda riga di annotazione è stato anche isolato il segmento corrispondente alla vocale precedente la seconda affricata dentale (tag "precedent vowel").

Una annotazione di questo tipo presuppone dunque una analisi di grana fine dei dati a disposizione, in linea con il paradigma sociofonetico scelto (cfr. Di Paolo \& Yaeger-Dror 2011). Tra tutti i fenomeni indagabili, in questa sede ci occuperemo principalmente del grado di sonorità, della durata e del luogo di articolazione delle affricate dentali, specificando che in quest'ultimo caso il luogo di articolazione verrà misurato solo per l'elemento fricativo.

Come già discusso in Meluzzi (2013), l'analisi spettroacustica ha evidenziato la presenza di tre gradi di sonorità: totalmente sordo, totalmente sonoro e intermedio. Quest'ultimo consta di una parte occlusiva dell'affricata in cui è chiaramente visibile la barra di sonorità, mentre tale sonorità scompare nel successivo elemento fricativo, che è anche uditivamente percepibile come sordo (cfr. Fig. 2). Nelle produzioni dei parlanti italofoni tale realizzazione si accompagna spesso a uno stacco (tag "E”) tra i due momenti occlusivo e fricativo dell'affricata dentale.

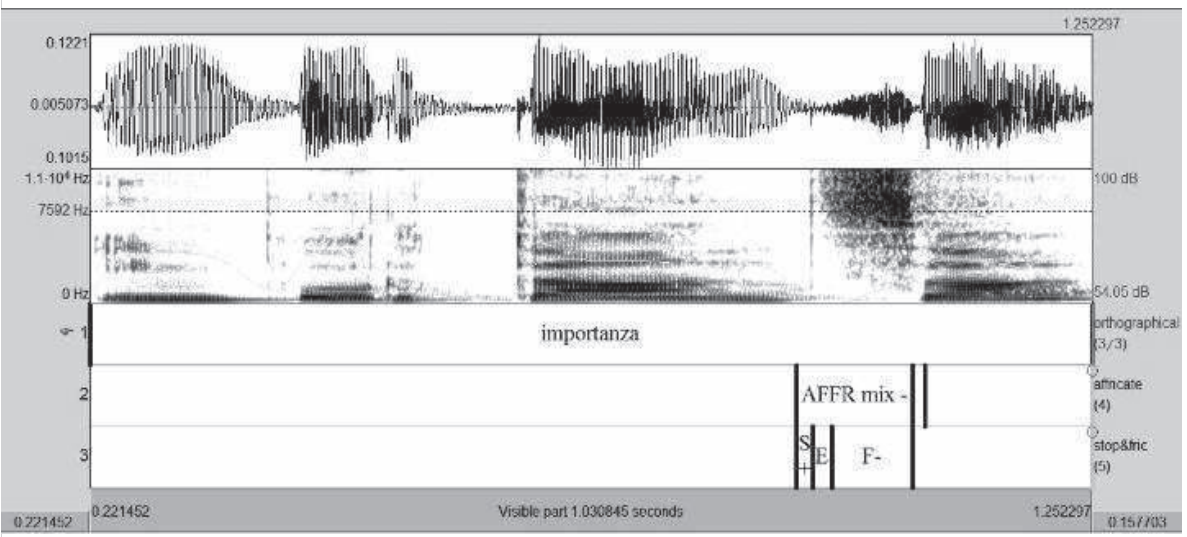

Fig. 2 Esempio di affricata intermedia per grado di sonorità (011StLG) 
"Zeta" di frontiera: confronto tra giovani bolzanini italofoni e germanofoni

C. MeLuzzI
Nel nostro corpus di giovani altoatesini, le affricate intermedie ricorrevano nel $24 \%$ in parlanti italofoni e nel $13,3 \%$ in parlanti tedescofoni (correlazione statisticamente significativa per $\mathrm{p}<0,001, \square^{2}=25,445$ ). Tuttavia le affricate intermedie dei tedescofoni presentavano spesso delle diversità dal punto di vista spettrografico, per esempio con una distribuzione della sonorità non uniforme in tutta la parte occlusiva dell'affricata dentale, come dimostra l'esempio in figura 3. Si può infatti notare una leggera sonorità iniziale, che però scompare ben prima del burst, risultando quindi in una affricata nel complesso percepita come completamente sorda. Questa sonorità iniziale può essere legata a una nasalizzazione, che si attesta tipicamente in fase di acquisizione ed è legata alla mancanza di sincronizzazione dei gesti in fase di produzione. $\mathrm{Si}$ tenga presente che il contrasto di sonorità è neutralizzato nel dialetto tedesco sudtirolese, ragion per cui è possibile qui ipotizzare anche una interferenza con la L1 della parlante. Il target di riferimento era dunque una affricata dentale sonora, ma la parlante L2 non ha gestito correttamente il tempo di produzione della sonorità, che si è dunque arrestata ben prima dello scoppio dell'affricata, risultando quindi in un target sordo, ma con questa leggera nasalizzazione iniziale.

Per questa ragione, in questa sede abbiamo preferito escludere dal nostro corpus tutti i casi di affricate dentali di grado intermedio, o di difficile classificazione come nell'esempio in figura 3, sia per il gruppo tedescofono sia, per bilanciamento, per il gruppo italofono, riservandoci una successiva analisi più puntuale di questi fenomeni in altra sede, eventualmente valutando anche l’impatto del grado di formalità del task a cui gli utenti erano stati sottoposti (appunto, una lettura di parole in isolamento). Il corpus è stato quindi ridotto a un numero totale di 428 occorrenze.

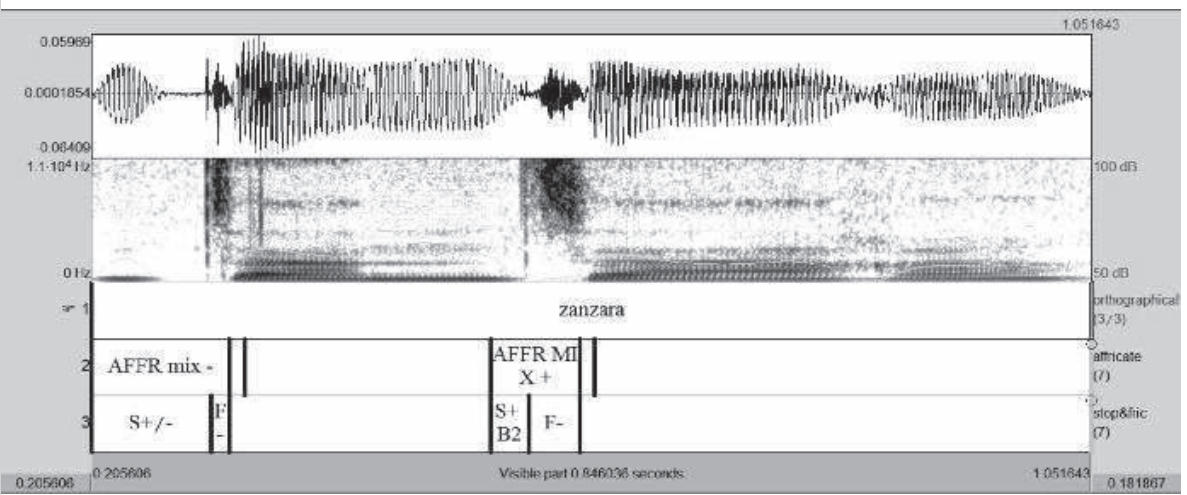

Fig. 3 Esempio di affricata intermedia pronunciata da un tedescofono (04LIS). 
"Zeta" di frontiera: confronto tra giovani bolzanini italofoni e germanofoni

C. MeluzzI
Riducendo quindi l'analisi del grado di sonorità alle due realizzazioni "canoniche" totalmente sorda o totalmente sonora, otteniamo una correlazione ancora statisticamente significativa $\left(p<0,001, \square^{2}=15,425\right)$ tra il tipo di realizzazione e il gruppo linguistico dei parlanti: in particolare, i tedescofoni realizzano con maggiore frequenza affricate sorde $(71,2 \%$ delle occorrenze) e non sonore (28,8\%), mentre gli italofoni realizzano quasi in egual misura foni sordi $(52,8 \%)$ e foni sonori $(47,2 \%)$, pur se con una leggera prevalenza dei primi sui secondi.

Risulta ovviamente interessante scorporare questo dato grezzo, andando a osservare la distribuzione del parametro di sonorità nei diversi contesti fonologici. La distribuzione, ancora una volta statisticamente significativa sia per il sottogruppo italiano $\left(\mathrm{p}<0,001, \square^{2}=42,883\right)$ sia per quello tedesco $\left(\mathrm{p}<0,001, \square^{2}=27,864\right)$, è riassunta nel grafico in figura 4.

Si può osservare come gli italofoni prediligano decisamente una resa sonora in contesto iniziale assoluto (\#C-), uniformandosi in questo a un modello di italiano settentrionale. I parlanti tedescofoni risultano fortemente influenzati da questo modello, tant'è che in questo contesto si registra più frequentemente una resa sonora (53,8\% dei casi), pur se la resa sorda resta molto frequente, contrastando quindi decisamente con il gruppo italofono. All'interno di parola, si può osservare come entrambi i contesti intervocalici (VCGV e VCV) registrino una distribuzione quasi omogenea del grado di sonorità nei due gruppi linguistici. Una differenza invece significativa si ritrova nel contesto post-sonorantico (SCV), nel quale i parlanti tedescofoni prediligono decisamente una resa sorda $(86,2 \%$ dei casi), laddove gli italofoni oscillano tra i due gradi di sonorità.

\begin{tabular}{|l|l|l|l|l|l|l|}
\hline & & \#C- & SCV & VCCV & VCV & TOT \\
\hline ITA & Sorda & 7058,4 & 8173,8 & 8209,2 & 8292,4 & 8104,51 \\
& & 0 & 5 & 0 & \\
\hline & Sonora & 8241,7 & 8723,11 & 7826,4 & 8323,9 & 8258,03 \\
& & 5 & & 6 & 5 & \\
\hline TED & Sorda & 8326,3 & 8109,2 & 8165,8 & 7942,0 & 8144,55 \\
& & 4 & 7 & 3 & 7 & \\
\hline & Sonora & 7984,3 & 7504,8 & 7972,0 & 7831,6 & 7907,04 \\
& & 1 & 9 & 6 & 5 & \\
\hline
\end{tabular}

Fig. 4 Distribuzione della sonorità nei diversi contesti fonologici nei due gruppi linguistici.

Questa distribuzione conferma come da un lato vi sia una influenza 
"Zeta" di frontiera: confronto tra giovani bolzanini italofoni e germanofoni

C. Meluzzı del modello di italiano L1, prevalentemente di tipo settentrionale, sul tipo di italiano L2 parlato dai tedescofoni altoatesini, pur se l'interferenza con la Ll rimane fortissima, portando quindi a realizzazioni tendenzialmente sorde in quasi tutti i contesti fonologici. D'altro canto, l'oscillazione tra sorda e sonora nei parlanti italofoni in contesto post-sonorantico riproduce una oscillazione tipica di questo fono tra i diversi italiani regionali (cfr. Loporcaro 2009, Meluzzi 2013: 46-50). Nonostante i parlanti analizzati siano giovani bolzanini, i cui genitori erano a loro volta nati in Alto Adige, nella loro pronuncia non vi è ancora una chiara tendenza verso l'una o l'altra realizzazione. Ciò mostra come l'italiano altoatesino non abbia ancora raggiunto una norma uniformemente condivisa, almeno per quanto riguarda il fono in analisi.

Per quanto riguarda invece la durata delle affricate dentali, il valore medio delle affricate dentali sonore di italofoni e tedescofoni risulta simile, attorno ai 108-109 msec, mentre le corrispettive sorde risultano leggermente più lunghe negli italofoni $(165 \mathrm{msec})$ rispetto ai tedescofoni $(162 \mathrm{msec})$. Bisogna tuttavia considerare che la velocità di eloquio, misurata sempre in millisecondi sulla parola target, risulta ovviamente diversa tra i due gruppi. Normalizzando i valori di durata mettendo in rapporto la durata dell'affricata dentale con la durata dell'intera parola, si ottiene tuttavia una distribuzione analoga, con una pronuncia quindi leggermente più lenta negli italofoni rispetto ai tedescofoni, specialmente nel caso delle affricate dentali sorde (25,56 contro 21,61).

In ogni caso, questi dati ci permettono di condurre alcune riflessioni in relazione a fenomeni analoghi registrati in altre varietà. Il fatto che $\mathrm{i}$ tedescofoni producano le affricate dentali italiane in un lasso di tempo minore rispetto ai madrelingua risulta infatti apparentemente sorprendente. Una spiegazione potrebbe forse venire da quanto evidenziato da Mossmüller (2014) rispetto al tedesco austriaco standard (Standard Austrian German, SAG). Nel campione analizzato dalla Mossmüller i giovani mostravano una netta tendenza per un eloquio più rapido, visibile in una riduzione della durata delle vocali rispetto a parlanti più anziani. In mancanza di dati analoghi sul tedesco altoatesino, un fenomeno analogo è quanto meno ipotizzabile come tendenza generale a una maggiore velocità di eloquio. Sarebbe interessante osservare contrastivamente questa differenza tra tedesco L1 e italiano L2 negli stessi parlanti, nonché in parlanti di età più avanzata.

Infine, per quanto riguarda il luogo di articolazione, sussiste anche in questo caso una differenza tra i due gruppi linguistici. Il valore del centro di gravità, misurato in $\mathrm{Hz}$, è direttamente proporzionale a un maggiore avanzamento nella realizzazione delle affricate dentali o almeno del loro elemento fricativo, sul quale è stata condotta l'indagine. Dai dati emerge come le affricate dentali sorde vengano realizzate circa con 
"Zeta" di frontiera: confronto tra giovani bolzanini italofoni e germanofoni

C. MeluzzI
CONCLUSIONI E PROSPETTIVE DI RICERCA lo stesso luogo da italiani e tedeschi, con una media del centro di gravità a $8104,51 \mathrm{~Hz}$ per i primi e a $8144,55 \mathrm{~Hz}$ per i secondi. La differenza maggiore tra i due gruppi linguistici si registra però per le affricate dentali sonore, con una media del centro di gravità di 8258,03 per il gruppo italofono e di 7907,0 per il gruppo tedescofono.

Rispetto ai diversi contesti fonologici, dai risultati presentati in tabella 2 si può notare come gli italiani mostrino quasi sempre valori medi del centro di gravità più alti dei tedescofoni, in particolare ancora una volta per quanto riguarda i foni sonori. Gli italiani dunque prediligono una pronuncia più avanzata. Questo può essere dovuto forse a un maggiore contatto, da parte degli italofoni, con l'italiano trentino, caratterizzato appunto da rese più avanzate, addirittura interdentali, dei foni fricativi. D'altro canto ancora una volta si può notare come le differenze tra i due gruppi linguistici siano rintracciabili in particolare nella resa delle affricate dentali sonore italiane, assente dal repertorio fonologico del tedesco.

\begin{tabular}{|l|l|l|l|l|l|l|}
\hline & & \#C- & SCV & VCCV & VCV & TOT \\
\hline ITA & Sorda & 7058,4 & 8173,8 & 8209,2 & 8292,4 & 8104,51 \\
& & 0 & 5 & 0 & \\
\hline & Sonora & 8241,7 & 8723,11 & 7826,4 & 8323,9 & 8258,03 \\
& & 5 & & 6 & 5 & \\
\hline TED & Sorda & 8326,3 & 8109,2 & 8165,8 & 7942,0 & 8144,55 \\
& & 4 & 7 & 3 & 7 & \\
\hline & Sonora & 7984,3 & 7504,8 & 7972,0 & 7831,6 & 7907,04 \\
& & 1 & 9 & 6 & 5 & \\
\hline
\end{tabular}

Tab. 2 Valori medi del centro di gravità (in $\mathrm{Hz}$ ) per gruppo linguistico, grado di sonorità e contesto fonologico.

Per l'analisi delle durate e del luogo di articolazione non è stato possibile effettuare un test di significatività statistica sulle medie. La distribuzione non normale dei dati nei nostri due sottocampioni, verificata tramite il test di normalità di Shapiro-Wilk per entrambe le variabili qui considerate, ci impedisce di verificare la significatività statistica di tali differenze attraverso un T-Test (cfr. Gray \& Kinnear 2012). D'altronde il ricorso a test di significatività per distribuzioni non normali non è uniformemente condiviso in campo statistico, specialmente in riferimento alle scienze sociali. Un ampliamento del campione potrà in questo senso giovare all'analisi statistica dei dati.

L'analisi della pronuncia delle affricate dentali italiane da parte di un gruppo di giovani altoatesini ha permesso di evidenziare alcuni fenomeni interessanti. In primo luogo si è potuta osservare una certa omogeneità delle realizzazioni sia in L1 sia in L2 per quanto riguarda la 
"Zeta" di frontiera: confronto tra giovani bolzanini italofoni e germanofoni

C. Meluzzı

\section{BIBLIOGRAFIA}

durata e il luogo di articolazione. Lievi differenze sono comunque rintracciabili all'interno dei due gruppi anche rispetto a questi parametri, lasciando avanzare l'ipotesi che siano in gioco due diversi modelli di riferimento: il tedesco austriaco per i tedescofoni e un generale italiano settentrionale, forse più legato al tipo trentino, per gli italofoni. I dati finora a disposizione sono però ancora decisamente parziali per poter permettere di avanzare affermazioni certe in proposito. Quanto emerge dai dati qui presentati deve andare quindi a costituire una ipotesi di lavoro per successivi sviluppi dell'indagine.

Dall'analisi del grado di articolazione è inoltre emersa una particolare preferenza dei tedescofoni per le realizzazioni sorde, in conformità quindi con quanto presente anche nel repertorio fonologico della propria L1. Si tratterebbe in questo caso di un esempio di influenza della L1 sulla L2, in cui, come scrivono Lléo et al. (2008: 189) "more complex and marked phenomena are learned with difficulty, require more time to be learned, and often are only learned in an incomplete manner". Infatti, l'affricata dentale sonora è un fono raro e marcato dal punto di vista tipologico (cfr. Maddieson 1984). Costamagna (2008) ha inoltre notato come proprio questo fono sia di difficile acquisizione per parlanti italiano L2 o LS.

Per quanto riguarda il gruppo italofono, inoltre, è possibile osservare come una varietà di italiano generalmente definibile come altoatesina è ancora di difficile definizione, data la grande variabilità registrabile anche all'interno di un gruppo di giovani parlanti italofoni.

Sarebbe ovviamente interessante confrontare gli stessi parlanti su altri foni, nonché specificare l'indagine, anche in chiave qualitativa, su quei fenomeni peculiari che sono emersi durante la fase di annotazione spettroacustica di questi foni. Dal punto di vista acquisizionale, potrebbe essere inoltre estremamente utile confrontare le produzioni dei tedescofoni nella propria L1, nonché del gruppo italofono in tedesco L2, al fine di poter verificare se e in che misura si istanzi una effettiva interferenza (cfr. Flege 1987) delle rispettive prime lingue sulle seconde.

ABEL A., C. VETTORI \& D. FORER. 2010. Learning the Neighbour's Language: the Many Challenges in Achieving a Real Multilingual Society. The Case of Second Language Acquisition in the Minority-Majority Context of South Tyrol, in European Centre for Minority Issues \& European Academy Bozen/Bolzano (Hrsgg.), European Yearbook of Minority Issues. Vol. 9. Leiden: Brill Academic Publishers.

ASTAT. 2012. Censimento della popolazione 2011. Città di Bolzano. BAUR S., G. MEZZALIRA \& W. PICHLER. 2008. La lingua degli altri. Aspetti della politica linguistica e scolastica in Alto Adige-Südtirol dal 1945 
"Zeta" di frontiera: confronto tra giovani bolzanini italofoni e germanofoni

C. MeluzzI ad oggi. Milano: Franco Angeli.

BOERSMA P. \& D. WEENINK. 2013. Praat: doing phonetics by computer [Computer program]. Version 5.3.51, retrieved 2 June 2013 from http://www.praat.org/.

COSTAMAGNA L. 2008. Affricates in Italian as L2: the role of psycho-attitudinal parameters, pp. 137-148 in L. Costamagna \& S. Giannini (a cura di). La fonologia dell'interlingua. Principi e metodi di analisi, Milano: Franco Angeli.

DI PAOLO M. \& M. YAEGER-DROR (eds). 2011. Sociophonetics. A student's guide. London/New York: Routledge.

FLEGE J. E. 1987. The production of 'new' and 'similar' phones in a foreign language: evidence for the effect of equivalence classification. fournal of Phonetics, $\mathrm{n}^{\circ}$ 15, 47-65.

FOULKES P., J. SCOBBIE \& D. WATT. 2010. Sociophonetics, pp. 703-716 in Laver J. e Gibbons F., Handbook of Phonetic Sciences, London, Blakcwell.

FREDDI G. 1982. Maggioranze, minoranze e plurilinguismo nella Provincia di Bolzano. Quaderni per la promozione del bilinguismo, n 31/32, 29-52.

GRAY G. D. \& P. R. KINNEAR. 2012. IBM SPSS 19 Statistics Made Simple. New York: Psychology Press.

LABOV W. 1994. Principles of linguistic change. Vol. 1: Internal factors. London, Blackwell.

LOPORGARO M. 2009. Profilo linguistico dei dialetti italiani. Roma/Bari: Laterza.

LUSCHÜTZKY H. C. 1992. Zur Phonologie der Affrikaten. Frankfurt am Main: Hector.

MADDIESON I. 1984. Patterns of sounds. Cambridge: Cambridge University Press.

MELUZZI C. 2013. Le affricate dentali nell'italiano di Bolzano. Un approccio sociofonetico. Tesi di dottorato.Università di Pavia / Libera Università di Bolzano.

MELUZZI C. 2014. "Italiano e Tedesco a Bolzano: la percezione degli italofoni", pp. 91-104 in A. De Meo, M. D’Agostino, G. Iannàccaro e L. Spreafico (eds). Varietà dei contesti di apprendimento linguistico. Studi AItLA 1.

MELUZZI C. 2015. Dialects and linguistic identity of Italian speakers in Bozen, Globe: a journal of language, culture and communication, 1, in stampa [online:

http://journals.aau.dk/index.php/globe/index].

PETRI R. 1989. Storia di Bolzano. Padova: Il Poligrafo. 